OPEN ACCESS

Edited by:

Mark P. Burns,

Georgetown University,

United States

Reviewed by:

Xuping Li,

Houston Methodist Research Institute, United States Zemin Wang,

Harvard Medical School, United States

*Correspondence: Chun-feng Liu

liuchunfeng@suda.edu.cn

${ }^{+}$Co-first authors and these authors have contributed equally to this work.

Specialty section:

This article was submitted to

Neurodegeneration,

a section of the journal

Frontiers in Neurology

Received: 14 August 2017 Accepted: 05 December 2017 Published: 05 January 2018

Citation:

Huang $H-j$, Zhu $X-y$, Wang $X$, Wang Z-y, Zhang W-I, Chen B-c and Liu C-f (2018) The Bulbocavernosus Reflex in the Differential Diagnosis of

Multiple System Atrophy with

Predominant Parkinsonism and

Parkinson's Disease.

Front. Neurol. 8:697.

doi: 10.3389/fneur.2017.00697

\section{The Bulbocavernosus Reflex in the Differential Diagnosis of Multiple System Atrophy with Predominant Parkinsonism and Parkinson's Disease}

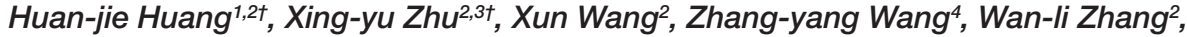 \\ Bi-cheng Chen ${ }^{5}$ and Chun-feng Liu ${ }^{1 *}$
}

${ }^{1}$ Department of Neurology, Suzhou Clinical Research Center of Neurological Disease, The Second Affiliated Hospital of Soochow University, Suzhou, China, ${ }^{2}$ Department of Neurology, The First Affiliated Hospital, Wenzhou Medical University, Wenzhou, China, ${ }^{3}$ School of the First Clinical Medical Sciences, Wenzhou Medical University, Wenzhou, China, ${ }^{4}$ Department of Neurology, Huashan Hospital, Shanghai Medical College, Fudan University, Shanghai, China, ${ }^{5}$ Zhejiang Provincial Top Key Discipline in Surgery, Wenzhou Key Laboratory of Surgery, Department of Surgery, The First Affiliated Hospital, Wenzhou Medical University, Wenzhou, China

Multiple system atrophy with predominant parkinsonism (MSA-P) is a degenerative disorder that presents with autonomic dysfunction, atypical parkinsonism, and ataxia. Parkinson's disease (PD) is an age-related neurological disorder of the central nervous system. Differentiation between MSA-P and PD is important because treatments, complications, and prognoses differ. The bulbocavernosus reflex (BCR) tests the afferent and efferent signals of the pudendal nerve as well as the sacral cord. In this study, we investigated differences in BCR parameters between MSA-P and PD patients. Thirty-eight MSA-P patients and 32 PD patients were selected to participate in our electrophysiological investigations. The Keypoint EMG/EP system was used to induce the BCR, and latencies and amplitudes were recorded for systematic statistical analyses. Area under the curve of the receiver operating characteristic was used to assess the specificity and sensitivity of the BCR parameters. A BCR was elicited in $76.32 \%$ of MSA-P patients and $93.75 \%$ of PD patients. The BCR latencies of the MSA-P group were longer than those of the PD group $(p<0.001)$. In addition, the MSA-P group had a lower BCR amplitude compared to the PD and control groups $(p<0.001)$. We discovered the difference between MSA-P and PD through BCR latencies and amplitudes. Compared to PD patients, MSA-P patients have longer latencies and lower amplitudes. Therefore, the BCR may be used to discriminate between MSA-P and PD in some cases.

Keywords: multiple system atrophy, Parkinson's disease, bulbocavernosus reflex, electrophysiological test, differential diagnosis

\section{INTRODUCTION}

The differentiation between Parkinson's disease (PD) and other parkinsonian diseases is a difficult issue in clinical diagnosis (1). The symptoms of multiple system atrophy with predominant parkinsonism (MSA-P) and PD are superimposed and similar. Ten percent of probable PD patients are eventually diagnosed with MSA (2). PD predominantly affects the motor system and 
sometimes influences the autonomic nervous system; however, MSA-P mainly affects the autonomic nervous system. When the autonomic nervous system is altered, constipation, bladder problems, and orthostatic hypotension often occur $(3,4)$. The parkinsonism paired with an autonomic nervous system disorder makes it hard to differentiate between MSA-P and PD patients (5). Different methods have been used to help make this discrimination and, of these, imaging methods have been the most popular (6). Other methods based on autonomic nervous system disorders have also been studied (7). Despite these previous attempts, an objective examination that includes the autonomic nervous system and that renders a greater possibility for differential diagnosis is still needed.

The bulbocavernosus reflex (BCR) causes the contraction of the bulbocavernosus muscle by stimulating the pudendal nerve, and this contraction can be recorded using the electromyogram technique. The BCR tests the afferent and efferent signals of the pudendal nerve as well as the sacral cord (8). A lesion of the reflex arc influences the BCR latencies and amplitudes. Over the last 20 years, the BCR has been used in the diagnosis of various neurogenic diseases, including neurogenic impotence, spinal shock, and cauda equine syndrome (9-11). The pudendal nerve somatosensory-evoked potential responses (PSEP) share the same pathway with the BCR before entering the spinal cord. Therefore, PSEP together with the BCR could be used to locate a lesion in the pathway. Previous research from our group suggests that an abnormality of BCR exists in MSA patients (12). Given that MSA and PD share the same symptoms of autonomic nervous system disorders, the abnormality may also exist in PD patients. In this study, we intend to distinguish between MSA-P and PD patients using the BCR measurement. Specifically, our study compared the latencies and amplitudes of the BCR and PSEP in 38 probable MSA-P patients, 32 PD patients, and 30 normal, healthy subjects. Statistical analyses were performed to differentiate between MSA-P and PD.

\section{MATERIALS AND METHODS}

\section{Subject}

In this study, 38 probable MSA-P patients and $32 \mathrm{PD}$ patients were selected to participate in the electrophysiological investigations between December 2012 and October 2014. All of the patients were referred to the First Affiliated Hospital of Wenzhou Medical University. All MSA-P patients met the inclusion criteria published in 2008 (13). Probable MSA-P patients were diagnosed by two inclusion criteria: autonomic failure involving urinary incontinence (inability to control the release of urine from the bladder, with erectile dysfunction in males) or an orthostatic decrease of blood pressure and poorly levodopa-responsive parkinsonism (bradykinesia with rigidity, tremor, or postural instability). Likewise, all PD patients met the Movement Disorder Society's clinical diagnostic criteria (14). PD patients were characterized by tremor at rest, rigidity, akinesia, and postural instability. In addition, all patients were evaluated under magnetic resonance imaging (MRI) and a follow-up visit. We also recruited 30 healthy subjects with no history of neurological or psychiatric diseases as a control group. All healthy controls underwent thorough clinical and neurologic examinations. Subjects with a history of parkinsonism, ataxia, autonomic dysfunction, or neurologic diseases (e.g., epilepsy or stroke) were excluded. Moreover, male subjects with prostatic diseases were excluded. MRI was used to exclude those subjects with cerebrovascular or space-occupying lesions.

All participants gave their written informed consent in accordance with the Declaration of Helsinki and agreed to participate in the study. This study was conducted under the approval of the Ethical Decision Committee of the Research Administration at the First Affiliated Hospital of Wenzhou Medical University (CR2009041).

\section{Electrophysiology}

The Keypoint EMG/EP system (Dantec, Bristol, UK) was used to conduct the BCR and PSEP tests. A ground electrode was placed against the thigh of patients in the lithotomy position. The temperature of the skin surface was maintained above $32^{\circ} \mathrm{C}$.

\section{Bulbocavernosus Reflex}

In males, ring-shaped stimulating electrodes were placed on the body or at the root of the penis. The positive pole was located at the corona glandis. In females, clamp-stimulating electrodes were placed near the pubic symphysis. The positive and negative poles were put on the clitoris and the labia vulvae, respectively. The BCR was recorded by concentric needle electrodes inserted into the scrotum at the perianal region near the midline. The stimulus intensity was seven times the individual's sensory threshold, and the electrode impedance was $<5 \mathrm{k} \Omega$. Stimulation of the square wave was 1.9 pulses/s, and we recorded the average value of 20 reflection waves. The scanning time was $5 \mathrm{~ms} /$ division, bandwidth range was $10 \mathrm{~Hz}$ to $2 \mathrm{kHz}$, and persistence time was $100 \mathrm{~ms}$. Latencies were calculated based on the beginning of the stimulus and the start of reflex response.

\section{Pudendal Nerve Somatosensory-Evoked Potential Responses}

The electrodes used to evoke pudendal nerve potentials were identical to those used to evoke the BCR, and they were applied to the same places. However, the intensity of the stimulus was three times the individual's sensory threshold. Similarly, the electrode impedance was maintained at $5 \mathrm{k} \Omega$. The frequency of the square wave was 5 pulses/s and was averaged across 200 waves. The scanning time was set to $0.2 \mathrm{~ms} /$ division, and the relevant persistence time was $100 \mathrm{~ms}$, with a bandwidth ranging from $10 \mathrm{~Hz}$ to $5 \mathrm{kHz}$. The recording electrode was located $2 \mathrm{~cm}$ behind $\mathrm{Cz}$, and the reference electrode was placed on the forehead at Fpz. The PSEP P41 wave was detected after stimulus delivery.

\section{Statistical Analysis}

The data were analyzed using the Statistical Package for the Social Sciences 22.0 (IBM, CA, USA) and Stata SE 12 (StataCorp, LP, USA). Normal distribution and homogeneity of variance were identified using the Kolmogorov-Smirnov test and Shapiro-Wilk test. A chi-square test was used to compare the elicitation rates 
of BCRs between the MSA-P, PD, and control groups. A one-way analysis of variance (ANOVA) was used to analyze the differences in the BCR parameters between the three groups (MSA-P, PD, and control) in males. PSEP parameters also analyzed with an ANOVA. A Bonferroni multiple comparison test was applied when significant differences were identified. This test revealed further contrasts between the groups. A Kruskal-Wallis rank test was used to compare the BCR and PSEP parameters between the three (MSA-P, PD, and control) in females. A Nemenyi test was applied to further evaluate the differences between each group. A receiver operating characteristic (ROC) analysis was used to evaluate the specificity and sensitivity of the BCR parameters. A value of $p \leq 0.05$ was considered statistically significant.

\section{RESULTS}

\section{General Patient Characteristics}

Thirty-eight probable MSA-P patients and 32 PD patients participated in the study. The age of the probable MSA-P patients ranged from 36 to 74 years, and the mean age was $54.21 \pm 7.92$ years. $39 \%$ of patients ( 15 cases) had erectile dysfunction, $45 \%$ of patients (17 cases) had urinary incontinence, and $76 \%$ of patients (22 cases) had orthostatic hypotension. In addition, the age of the diagnosed PD patients ranged from 35 to 77 years, and the mean age was $55.23 \pm 8.75$ years. $22 \%$ of patients ( 7 cases) had erectile dysfunction, $3 \%$ of patients ( 1 case) had urinary incontinence, and $16 \%$ of patients (5 cases) had orthostatic hypotension. Both the MSA-P and PD groups suffered from autonomic failure (see Table 1).

\section{BCR Measurements}

Elicitation rates in the MSA-P, PD, and control groups were $76.3 \%$ (29/38), 93.75\% (30/32), and 100\% (30/30). There was a significant difference in the BCR elicitation rates between the three groups $\left(\chi^{2}=10.01, p=0.003\right)$. The BCR latencies in the MSA-P group were longer than those of the control and PD groups. There was no significant difference between the $\mathrm{PD}$ and control group latencies

TABLE 1 | General characteristics and elicitation rates of the bulbocavernosus reflex $(\mathrm{BCR})$ latencies between multiple system atrophy with predominant parkinsonism (MSA-P), Parkinson's disease (PD), and control groups.

\begin{tabular}{|c|c|c|c|c|}
\hline & & MSA-P & PD & Control \\
\hline \multirow[t]{2}{*}{ Ages } & Mean & $54.21 \pm 7.92$ & $55.23 \pm 8.75$ & $57.16 \pm 7.25$ \\
\hline & Ranges & 36-74 & 35-77 & 38-70 \\
\hline \multicolumn{2}{|c|}{ Males } & 18 & 17 & 14 \\
\hline \multicolumn{2}{|c|}{ Females } & 20 & 15 & 16 \\
\hline \multirow[t]{3}{*}{ Total } & & 38 & 32 & 30 \\
\hline & Duration (month) & 26.4 & 46.5 & - \\
\hline & Range & $4-72$ & $6-240$ & - \\
\hline \multicolumn{2}{|c|}{ Erectile dysfunction } & 15 & 7 & - \\
\hline \multicolumn{2}{|c|}{ Urinary incontinence } & 17 & 1 & - \\
\hline \multicolumn{2}{|c|}{ Orthostatic hypotension } & 22 & 5 & - \\
\hline \multicolumn{2}{|c|}{ Available for $\mathrm{BCR}$} & 29 & 30 & 30 \\
\hline \multicolumn{2}{|c|}{ Elicitation rates of $\mathrm{BCR}^{\mathrm{a}}$} & $76.32 \%$ & $93.75 \%$ & $100 \%$ \\
\hline
\end{tabular}

${ }^{a} \chi^{2}=10.01, p=0.003$ in either gender ( $p=1.000$ in male, $p=0.806$ left, $p=0.902$ right in female, see Table 2; Figures 1A,B). The BCR amplitudes of the MSA-P, PD, and control groups were significantly different from each other ( $p<0.01$, see Table 3; Figures 1C,D). The MSA-P group had the lowest amplitudes, and the control group had the highest amplitudes. In ROC curve, significant differences were found between the groups (area under the curve $=1.000$ ).

\section{PSEP Measurements}

No significant differences existed between the MSA-P, PD, and control groups in either the males or females (see Table 4).

\section{DISCUSSION}

In this study, we discovered that a difference found in BCR parameters could help to distinguish between MSA-P and PD. MSA-P patients exhibited a lower BCR elicitation rate than did PD patients (76.3 vs $90.9 \%$ ). The BCR parameters revealed differences between the MSA-P, PD, and control groups. MSA-P patients exhibited longer latencies and lower amplitudes than did the PD group. In addition, both of these parameters could significantly distinguish MSA-P using an ROC curve.

The afferent somatic sensory pathway of the BCR includes the pudendal nerve. The efferent somatic motor pathway is composed of the spinal segments S2-S4, including Onuf's nucleus and the pudendal nerve. An impulse contracts the bulbocavernosus muscle $(8,15)$. The afferent somatic sensory pathway of PSEPs comprises two segments, and the anterior segment shares the same afferent pathway with the BCR. An impulse is then transmitted to the cerebral cortex through the spinal cord. The combination of the BCR and PSEPs could be used to locate a lesion in some neuropathies, such as diabetic neurogenic bladder and cauda equine syndrome.

In this study, MSA-P and PD patients had an abnormal BCR and normal PSEPs. The elicitation rates of MSA-P patients were lower than those of $\mathrm{PD}$ patients. In addition, we failed to elicit the BCR in $23.68 \%$ of the MSA-P patients. The lesion of reflex arc in the BCR of the MSA-P patients was more serious than in the PD patients. Furthermore, MSA-P patients exhibited

TABLE 2 | Comparison of the mean or median bulbocavernosus reflex latencies between multiple system atrophy with predominant parkinsonism (MSA-P), Parkinson's disease (PD), and control groups.

\begin{tabular}{|c|c|c|c|c|}
\hline & \multicolumn{2}{|c|}{ Latencies of males (ms) } & \multicolumn{2}{|c|}{ Latencies of females (ms) } \\
\hline & Left & Right & Left & Right \\
\hline MSA-P & $62.843 \pm 1.136$ & $63.221 \pm 1.172$ & $69.647 \pm 1.461$ & $69.827 \pm 1.461$ \\
\hline PD & $34.780 \pm 5.234$ & $34.793 \pm 4.939$ & $37.600 \pm 6.255$ & $37.393 \pm 6.215$ \\
\hline Control & $\begin{array}{c}33.800 \pm 5.022 \\
F=162.613 \\
p<0.001\end{array}$ & $\begin{array}{c}34.243 \pm 5.142 \\
F=166.245 \\
p<0.001\end{array}$ & $\begin{array}{c}36.313 \pm 6.048 \\
H=30.122 \\
p<0.001\end{array}$ & $\begin{array}{c}36.475 \pm 6.180 \\
H=29.893 \\
p<0.001\end{array}$ \\
\hline
\end{tabular}

Male.

MSA-P vs Control, PD vs MSA-P $p<0.001, P D$ vs Control $p=1.000$.

Female (Left, Nemeyi test).

$P D$ vs Control $p=0.806, M S A-P$ vs Control, MSA-P vs $P D p<0.001$.

Female (Right, Nemeyi test).

$P D$ vs Control $p=0.902$, MSA-P vs Control, MSA-P vs $P D p<0.001$. 

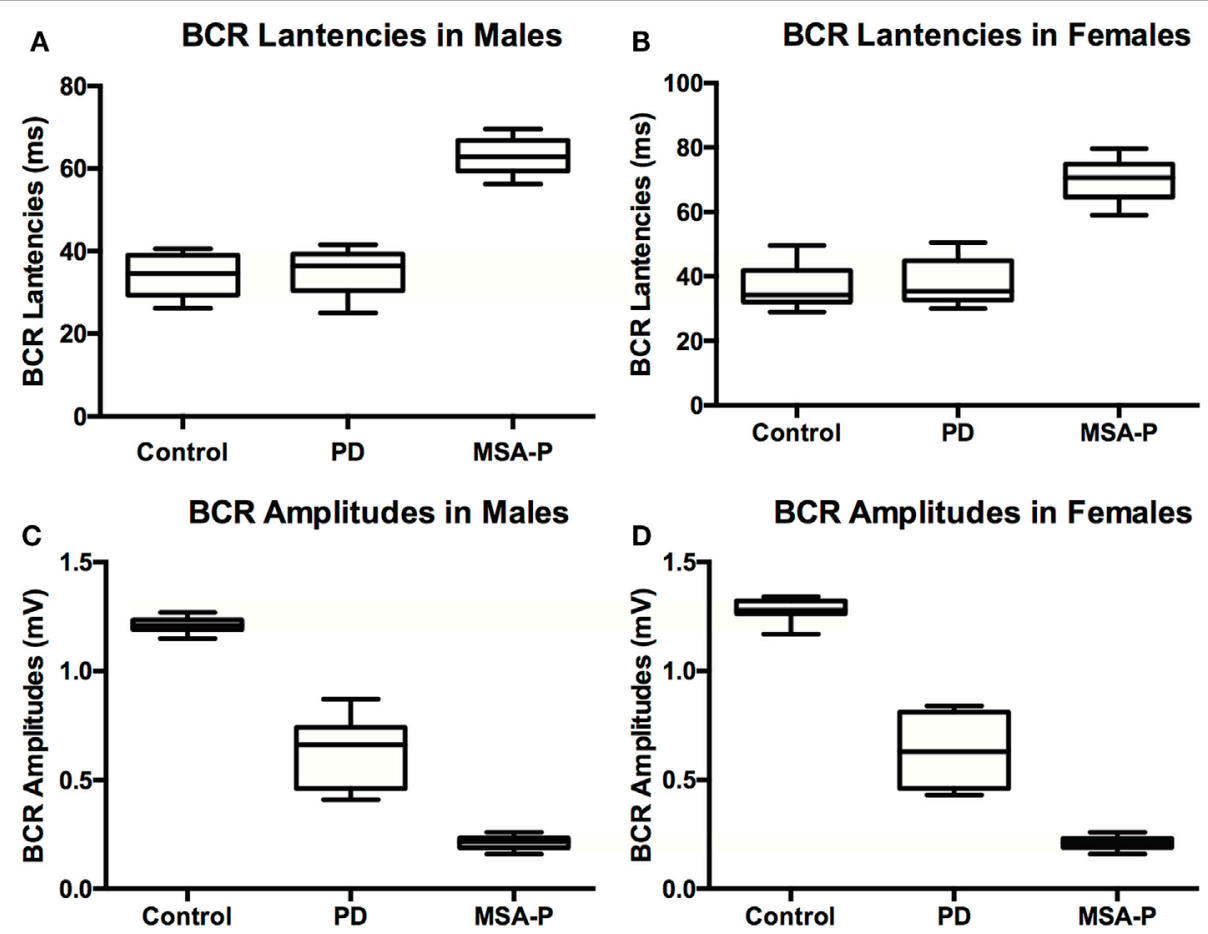

FIGURE 1 | Bulbocavernosus reflex (BCR) latencies and amplitudes between multiple system atrophy with predominant parkinsonism (MSA-P), Parkinson's disease (PD), and control groups in males (A) and females (B). BCR latencies were not significantly different between control and PD patients, but MSA patients had prolonged latencies compared to the other groups in males (C) and females (D). BCR amplitudes were significantly lower in MSA-P patients than those in the control group, and amplitudes of PD patients fell in between control and MSA-P amplitudes.

TABLE 3 | Comparison of the mean or median bulbocavernosus reflex amplitudes between multiple system atrophy with predominant parkinsonism (MSA-P), Parkinson's disease (PD), and control groups.

\begin{tabular}{|c|c|c|c|c|}
\hline & \multicolumn{2}{|c|}{ Amplitudes of males (mV) } & \multicolumn{2}{|c|}{ Amplitudes of females (mV) } \\
\hline & Left & Right & Left & Right \\
\hline MSA-P & $0.209 \pm 0.008$ & $0.207 \pm 0.007$ & $0.207 \pm 0.008$ & $0.205 \pm 0.009$ \\
\hline PD & $0.631 \pm 0.156$ & $0.625 \pm 0.152$ & $0.631 \pm 0.156$ & $0.625 \pm 0.152$ \\
\hline Control & $\begin{array}{c}1.213 \pm 0.033 \\
F=387.214 \\
p<0.001\end{array}$ & $\begin{array}{c}1.210 \pm 0.032 \\
F=412.274 \\
p<0.001\end{array}$ & $\begin{array}{c}1.286 \pm 0.046 \\
H=40.047 \\
p<0.001\end{array}$ & $\begin{array}{c}1.272 \pm 0.042 \\
H=40.064 \\
p<0.001\end{array}$ \\
\hline
\end{tabular}

Male.

MSA-P vs Control, PD vs Control, PD vs MSA-P, $p<0.001$

Female (Left and Right, Nemeyi test).

$P D$ vs Control $p=0.006, M S A-P$ vs Control $p=0.009, M S A-P$ vs $P D p<0.001$.

lower BCR amplitudes than did PD patients. However, the PSEPs revealed no differences between the MSA-P, PD, and control subjects. The PSEP data cannot be used to distinguish between MSA-P and PD patients in our study. The pathway of PSEPs seemed to be complete in both the MSA-P and PD patients. As the BCR and PSEPs share the same afferent pathway, the lesions are likely to be located on the efferent pathway, which consists of the spinal segments S2-S4, including Onuf's nucleus, or on the pudendal nerve. These findings support the results of several previous studies. Konno et al. (16) found deprivation loss of somatic motor neurons in Onuf's nucleus. Moreover, the previous studies of Ref. (17) have found that
TABLE 4 | Comparison of the mean pudendal nerve somatosensory-evoked potential responses P41 latencies and amplitudes between multiple system atrophy with predominant parkinsonism (MSA-P), Parkinson's disease (PD), and control groups.

\begin{tabular}{|c|c|c|c|c|}
\hline & \multicolumn{2}{|c|}{ Males } & \multicolumn{2}{|c|}{ Females } \\
\hline & $\begin{array}{l}\text { Latencies } \\
\text { (ms) }\end{array}$ & $\begin{array}{c}\text { Amplitudes } \\
(\mathrm{mV})\end{array}$ & $\begin{array}{l}\text { Latencies } \\
\text { (ms) }\end{array}$ & $\begin{array}{c}\text { Amplitudes } \\
\text { (mV) }\end{array}$ \\
\hline MSA-P & $42.911 \pm 0.659$ & $2.684 \pm 0.065$ & $42.067 \pm 0.643$ & $2.626 \pm 0.083$ \\
\hline PD & $41.480 \pm 2.944$ & $2.765 \pm 0.286$ & $43.087 \pm 2.986$ & $2.672 \pm 0.394$ \\
\hline Control & $\begin{array}{c}40.629 \pm 1.931 \\
H=5.308 \\
p=0.070\end{array}$ & $\begin{array}{c}2.650 \pm 0.330 \\
H=0.595 \\
p=0.556\end{array}$ & $\begin{array}{c}44.238 \pm 2.256 \\
H=5.695 \\
p=0.058\end{array}$ & $\begin{array}{c}2.654 \pm 0.344 \\
H=0.218 \\
p=0.897\end{array}$ \\
\hline
\end{tabular}

the external anal sphincter electromyography (EAS-EMG) shares the same pathway with BCR. In addition, their results also show the degeneration of Onuf's nucleus anterior horn cell. According to these studies, the lesion in the BCR is likely positioned in Onuf's nucleus. It is also important to note that compared to the EAS-EMG, the methods used in our study are non-invasive.

Parkinson's disease is characterized by motor symptoms, such as resting tremor, rigidity, and slowness of movement, which can all be explained by the degeneration of neurons in the central nervous system. However, the non-motor-related symptoms in PD patients, such as constipation, bladder problems, and orthostatic hypotension have recently gained increasing attention. In this study, we found that the BCR amplitudes were 
lower than those in the control group, but higher than those in the MSA-P group. This suggests that certain lesions most likely involve the BCR in PD patients, but this condition is more serious in MSA-P patients. The autonomic nervous system is more seriously affected in MSA-P patients.

Shared non-motor symptoms in PD and MSA-P patients make it difficult to differentially diagnose MSA-P and PD patients, especially at an early stage. Multiple tests have been used in the last few decades in an attempt to solve this problem. Imaging methods, such as positron emission tomography, diffusion weighted image, and diffusion tensor image, have been used previously (18-20). The application of MRI does improve clinical accuracy $(6,21)$. Moreover, $3 \mathrm{~T}$ susceptibility-weighted imaging is more susceptible to putaminal changes and lesion asymmetry (21). Using brain volumetry with MRI is inefficient sometimes (22). In mildly symptomatic PD and MSA-P patients, brain perfusion is altered through the single-photon emission computed tomography method (23). However, with this technique, some influencing factor could disturb the results, and the process of data analysis is complex and time-consuming. In addition, the cost of radiological examination should also be considered, especially in developing countries. Other methods, such as measuring cerebrospinal fluid levels lack accuracy $(24,25)$. The examination of autonomic nerve function has become a research focus in recent years $(7,26,27)$. This method consists of a battery of cardiovascular autonomic tests, including orthostatic hypotension, systolic and diastolic blood pressure, and other tests. Using this method, Pilleri et al. (28) found that MSA patients exhibited increased nocturnal heart rate and reduced nocturnal decline of heart rate compared to PD patients. Pros and cons exist in the methods above; no single test can have both favorable sensitivity and specificity. An objective examination renders a greater possibility for accurate differential diagnosis.

Our study has some limitations. For example, the sample population of our study was restricted to available patients. In addition, all patients with MSA-P and PD were diagnosed clinically rather than pathologically.

\section{REFERENCES}

1. Wenning GK, Stefanova N. Recent developments in multiple system atrophy. J Neurol (2009) 256(11):1791-808. doi:10.1007/s00415-009-5173-8

2. Hughes AJ, Daniel SE, Kilford L, Lees AJ. Accuracy of clinical diagnosis of idiopathic Parkinson's disease: a clinico-pathological study of 100 cases. J Neurol Neurosurg Psychiatry (1992) 55(3):181-4. doi:10.1136/jnnp. 55.3.181

3. Farrer MJ. Genetics of Parkinson disease: paradigm shifts and future prospects. Nat Rev Genet (2006) 7(4):306-18. doi:10.1038/nrg1831

4. Schapira AH, Tolosa E. Molecular and clinical prodrome of Parkinson disease: implications for treatment. Nat Rev Neurol (2010) 6(6):309-17. doi:10.1038/ nrneurol.2010.52

5. Terao Y, Fukuda H, Tokushige S, Inomata-Terada S, Yugeta A, Hamada M, et al. Is multiple system atrophy with cerebellar ataxia (MSA-C) like spinocerebellar ataxia and multiple system atrophy with parkinsonism (MSA-P) like Parkinson's disease? A saccade study on pathophysiology. Clin Neurophysiol (2016) 127(2):1491-502. doi:10.1016/j.clinph.2015.07.035

6. Ramli N, Nair SR, Ramli NM, Lim SY. Differentiating multiple-system atrophy from Parkinson's disease. Clin Radiol (2015) 70(5):555-64. doi:10.1016/j. crad.2015.01.005
In conclusion, we observed a difference between MSA-P and PD patients in the BCR. Moreover, we consider the BCR test to be a non-invasive, convincing, objective, and economic approach to discriminate between MSA-P and PD patients. Supplementary study with more cases will validate our findings. Further application including the trace of BCR alteration with the progression of MSA-P or the association between BCR parameters and prognosis may help us better monitor these devastating neurological diseases.

\section{ETHICS STATEMENT}

All participants gave their written informed consent in accordance with the Declaration of Helsinki and agreed to participate in the study. This study was conducted under the approval of the Ethical Decision Committee of the Research Administration at the First Affiliated Hospital of Wenzhou Medical University (CR2009041).

\section{AUTHOR CONTRIBUTIONS}

$\mathrm{H}-\mathrm{jH}$ and $\mathrm{X}-\mathrm{yZ}$ analyzed the data, wrote and revised the article. $\mathrm{Z}-\mathrm{yW}$ participated in the writing and revised the article. XW and $\mathrm{W}-\mathrm{lZ}$ performed the clinical tests and collected the data. $\mathrm{B}-\mathrm{cC}$ and $\mathrm{C}$-fL conceived and designed the study and obtained ethics approval.

\section{ACKNOWLEDGMENTS}

We gratefully acknowledge Feng-ming Zhang from the School of the First Clinical Medical Sciences, Wenzhou Medical University, for assistance with the experiment and acquiring funding.

\section{FUNDING}

This work was funded by the Undergraduate Innovation Project of WMU (No. wyx2016101006).

7. Iodice V, Low DA, Vichayanrat E, Mathias CJ. Cardiovascular autonomic dysfunction in MSA and Parkinson's disease: similarities and differences. J Neurol Sci (2011) 310(1-2):133-8. doi:10.1016/j.jns.2011.07.014

8. Skinner SA, Vodusek DB. Intraoperative recording of the bulbocavernosus reflex. JClin Neurophysiol (2014) 31(4):313-22. doi:10.1097/WNP. 0000000000000054

9. Ashraf VV, Taly AB, Nair KP, Rao S, Sridhar. Role of clinical neurophysiological tests in evaluation of erectile dysfunction in people with spinal cord disorders. Neurol India (2005) 53(1):32-5; discussion 35-6. doi:10.4103/0028-3886.15048

10. Niu X, Shao B, Ni P, Wang X, Chen X, Zhu B, et al. Bulbocavernosus reflex and pudendal nerve somatosensory-evoked potentials responses in female patients with nerve system diseases. J Clin Neurophysiol (2010) 27(3):207-11. doi:10.1097/WNP.0b013e3181dd4fca

11. Laudano MA, Chughtai B, Lee RK, Seklehner S, Elterman D, Kaplan SA, et al. Use of the bulbocavernosus reflex system in assessing voiding dysfunction. World J Urol (2013) 31(6):1459-62. doi:10.1007/s00345013-1063-X

12. Wang ZY, Chen YH, Xu YY, Wang X, Shao B, Niu XT, et al. Altered bulbocavernosus reflex in patients with multiple system atrophy. Neurol Res (2016) 38(2):138-43. doi:10.1080/01616412.2015.1115222 
13. Gilman S, Wenning GK, Low PA, Brooks DJ, Mathias CJ, Trojanowski JQ, et al. Second consensus statement on the diagnosis of multiple system atrophy. Neurology (2008) 71(9):670-6. doi:10.1212/01.wnl.0000324625.00404.15

14. Postuma RB, Berg D, Stern M, Poewe W, Olanow CW, Oertel W, et al. MDS clinical diagnostic criteria for Parkinson's disease. Mov Disord (2015) 30(12):1591-601. doi:10.1002/mds.26424

15. Granata G, Padua L, Rossi F, De Franco P, CoraciD, Rossi V.Electrophysiological study of the bulbocavernosus reflex: normative data. Funct Neurol (2013) 28(4):293-5. doi:10.11138/FNeur/2013.28.4.293

16. Konno H, Yamamoto T, Iwasaki Y, Iizuka H. Shy-Drager syndrome and amyotrophic lateral sclerosis. Cytoarchitectonic and morphometric studies of sacral autonomic neurons. J Neurol Sci (1986) 73(2):193-204. doi:10.1016/0022-510x(86)90130-9

17. Han W, Cui LY, Hua D, Li BH. Relationship between the autonomic dysfunction of multiple system atrophy and external anal sphincter electromyograph. Chin J Neurol (2006) 2:109-12. doi:10.3760/j.issn:1006-7876. 2006.02.010

18. Wu-lin W, Xiao-yi W, Ming-wu L, Wei-hua L. DWI in differential diagnosis between multiple system atrophy and Parkinson disease. Chin J Imaging Technol (2009) 6:981-4. doi:10.3321/j.issn:1003-3289.2009.06.018

19. Cnyrim CD, Kupsch A, Ebersbach G, Hoffmann KT. Diffusion tensor imaging in idiopathic Parkinson's disease and multisystem atrophy (parkinsonian type). Neurodegener Dis (2014) 13(1):1-8. doi:10.1159/000348512

20. Sako W, Abe T, Murakami N, Miyazaki Y, Izumi Y, Harada M, et al. Imaging-based differential diagnosis between multiple system atrophy and Parkinson's disease. J Neurol Sci (2016) 368:104-8. doi:10.1016/j.jns. 2016.06.061

21. Hwang I, Sohn CH, Kang KM, Jeon BS, Kim HJ, Choi SH, et al. Differentiation of parkinsonism-predominant multiple system atrophy from idiopathic Parkinson disease using 3T susceptibility-weighted MR imaging, focusing on putaminal change and lesion asymmetry. AJNR Am J Neuroradiol (2015) 36(12):2227-34. doi:10.3174/ajnr.A4442

22. Huppertz HJ, Moller L, Sudmeyer M, Hilker R, Hattingen E, Egger K, et al. Differentiation of neurodegenerative parkinsonian syndromes by volumetric magnetic resonance imaging analysis and support vector machine classification. Mov Disord (2016) 31(10):1506-17. doi:10.1002/mds.26715
23. Song IU, Yoo I, Chung YA, Jeong J. The value of brain perfusion SPECT for differentiation between mildly symptomatic idiopathic Parkinson's disease and the Parkinson variant of multiple system atrophy. Nucl Med Commun (2015) 36(10):1049-54. doi:10.1097/MNM.0000000000000354

24. Ghaemi M, Hilker R, Rudolf J, Sobesky J, Heiss WD. Differentiating multiple system atrophy from Parkinson's disease: contribution of striatal and midbrain MRI volumetry and multi-tracer PET imaging. J Neurol Neurosurg Psychiatry (2002) 73(5):517-23. doi:10.1136/jnnp.73.5.517

25. Herbert MK, Eeftens JM, Aerts MB, Esselink RA, Bloem BR, Kuiperij HB, et al. CSF levels of DJ-1 and tau distinguish MSA patients from PD patients and controls. Parkinsonism Relat Disord (2014) 20(1):112-5. doi:10.1016/j. parkreldis.2013.09.003

26. Baschieri F, Calandra-Buonaura G, Doria A, Mastrolilli F, Palareti A, Barletta G, et al. Cardiovascular autonomic testing performed with a new integrated instrumental approach is useful in differentiating MSA-P from PD at an early stage. Parkinsonism Relat Disord (2015) 21(5):477-82. doi:10.1016/j.parkreldis.2015.02.011

27. Roy S, Srivastava AK, Jaryal AK, Deepak KK. Cardiovascular responses during cold pressor test are different in Parkinson disease and multiple system atrophy with parkinsonism. Clin Auton Res (2015) 25(4):219-24. doi:10.1007/ s10286-015-0294-x

28. Pilleri M, Levedianos G, Weis L, Gasparoli E, Facchini S, Biundo R, et al. Heart rate circadian profile in the differential diagnosis between Parkinson disease and multiple system atrophy. Parkinsonism Relat Disord (2014) 20(2):217-21. doi:10.1016/j.parkreldis.2013.11.006

Conflict of Interest Statement: The authors declare that the research was conducted in the absence of any commercial or financial relationships that could be construed as a potential conflict of interest.

Copyright (c) 2018 Huang, Zhu, Wang, Wang, Zhang, Chen and Liu. This is an open-access article distributed under the terms of the Creative Commons Attribution License (CC BY). The use, distribution or reproduction in other forums is permitted, provided the original author(s) or licensor are credited and that the original publication in this journal is cited, in accordance with accepted academic practice. No use, distribution or reproduction is permitted which does not comply with these terms. 